\title{
Autochthonous Emergomyces pasteurianus pneumonia in an immunocompromised patient in Hong Kong: a case report
}

\author{
KK Chik *, FHKPath, FHKPaed, WK To, FHKPath \\ Department of Pathology (Clinical Infection and Microbiology), Princess Margaret Hospital, Hong Kong \\ *Corresponding author: chikkk@ha.org.hk \\ Hong Kong Med J 2020;26:446-8 \\ https://doi.org/10.12809/hkmj198280
}

\section{Case}

A 61-year-old man with end-stage renal failure secondary to immunoglobulin A nephropathy underwent a cadaveric kidney transplant. His posttransplantation course was uneventful until he had very poor compliance to the immunosuppressants since May 2017 and admitted that he had stopped all immunosuppressants in February 2018. He developed acute antibody-mediated graft rejection in May 2018. His medications were adjusted, tacrolimus $4 \mathrm{mg} /$ day, mycophenolate $360 \mathrm{mg}$ twice daily and prednisolone were started. He developed a chest infection in October 2018. He denied any travel history or significant contact history. On physical examination he had cushingoid features and right-sided crepitation. Investigations revealed a low white cell count $\left(1.8 \times 10^{9} / \mathrm{L}\right)$ and neutrophil count $\left(0.7 \times 10^{9} / \mathrm{L}\right)$. Chest X-ray showed right basal infiltrates (Fig 1a). Blood and sputum for bacterial culture was negative, as was sputum for acid-fast bacilli. The patient's condition deteriorated despite use of vancomycin, meropenem, azithromycin, and fluconazole. Computed tomography of the thorax revealed extensive collapse and consolidation over the right lower lobe and bilateral pleural effusions (Fig 1b). Bronchoscopy and transbronchial lung biopsy showed granulomatous inflammation, with granular eosinophilic material and narrowbased small budding yeasts grouped in clusters inside macrophages in the alveolar spaces. Both mucicarmine staining and immunohistochemical staining for Pneumocystis, Cytomegalovirus, and herpes simplex virus were negative (Fig 1d-g). In view of the histopathological findings, bronchoalveolar lavage was sent for fungal culture. After 7 days of incubation at $25^{\circ} \mathrm{C}$, a tiny colony of mould was seen. 21 Days later, there was a white-coloured mould colony with a velvety texture, wrinkled surface, acquired splits on the surface with no diffusible pigment (Fig $2 \mathrm{a}$ and $\mathrm{b}$ ). Lactophenol cotton blue stain of the wet mount revealed a classic floret pattern (Fig 2c and d). Subculture was performed at $35^{\circ} \mathrm{C}$. After 10 days of incubation at $35^{\circ} \mathrm{C}$, a 25 - $\mathrm{mm}$-diameter yeast colony was evident. Based on the characteristic growth morphology, infection with the thermally dimorphic fungus was established. Subsequent molecular genetic analysis by sequencing of the internal transcribed spacer and D1/D2 regions was performed and identified Emergomyces pasteurianus, a rare thermally dimorphic fungus not previously reported in our locality. Liposomal amphotericin B was commenced and continued for 8 weeks. The patient responded well both clinically and radiologically (Fig 1c) and treatment was switched to oral voriconazole $200 \mathrm{mg}$ twice daily. The patient succumbed to his medical illness 10 weeks after being discharged home.

\section{Discussion}

Thermally dimorphic fungal pathogens cause a significant human disease but are rarely reported in our locality with the exception of Talaromyces (Penicillium) marneffei. To the best of our knowledge, this is the first report of Emergomyces infection in Hong Kong. Emergomyces shares the characteristics of other thermally dimorphic fungi: filamentous forms at $25^{\circ} \mathrm{C}$ that becomes an invasive yeast-like form at $35^{\circ} \mathrm{C} .{ }^{1}$ Emergomyces pasteurianus was previously known as Emmonsia pasteuriana. Emmonsia species are ubiquitous, soil-dwelling saprophytic fungi. Species such as Emmonsia crescens and Emmonsia parva may rarely cause adiaspiromycosis in rodents and humans. ${ }^{2}$ Recently, Emergomyces has been reclassified as a new genus within the family Ajellomycetaceae. Emergomyces and Emmonsia have significant differences in microbiology, epidemiology, clinical manifestations, and treatment outcomes. Microbiologically, Emergomyces is a thermally dimorphic fungus while Emmonsia does not undergo mould-to-yeast conversion at $37^{\circ} \mathrm{C}$. Emmonsia is rarely cultivated from clinical specimens. Human infection with Emmonsia is relatively rare in clinical practice ${ }^{2}$ but Emergomyces can cause fatal and disseminated human infection and appropriate antifungal therapy is essential.

At the time of this report, five Emergomyces species are described. These species differ in geographic distribution. Es pasteurianus has been reported in Europe, Asia, and Africa. Emergomyces 


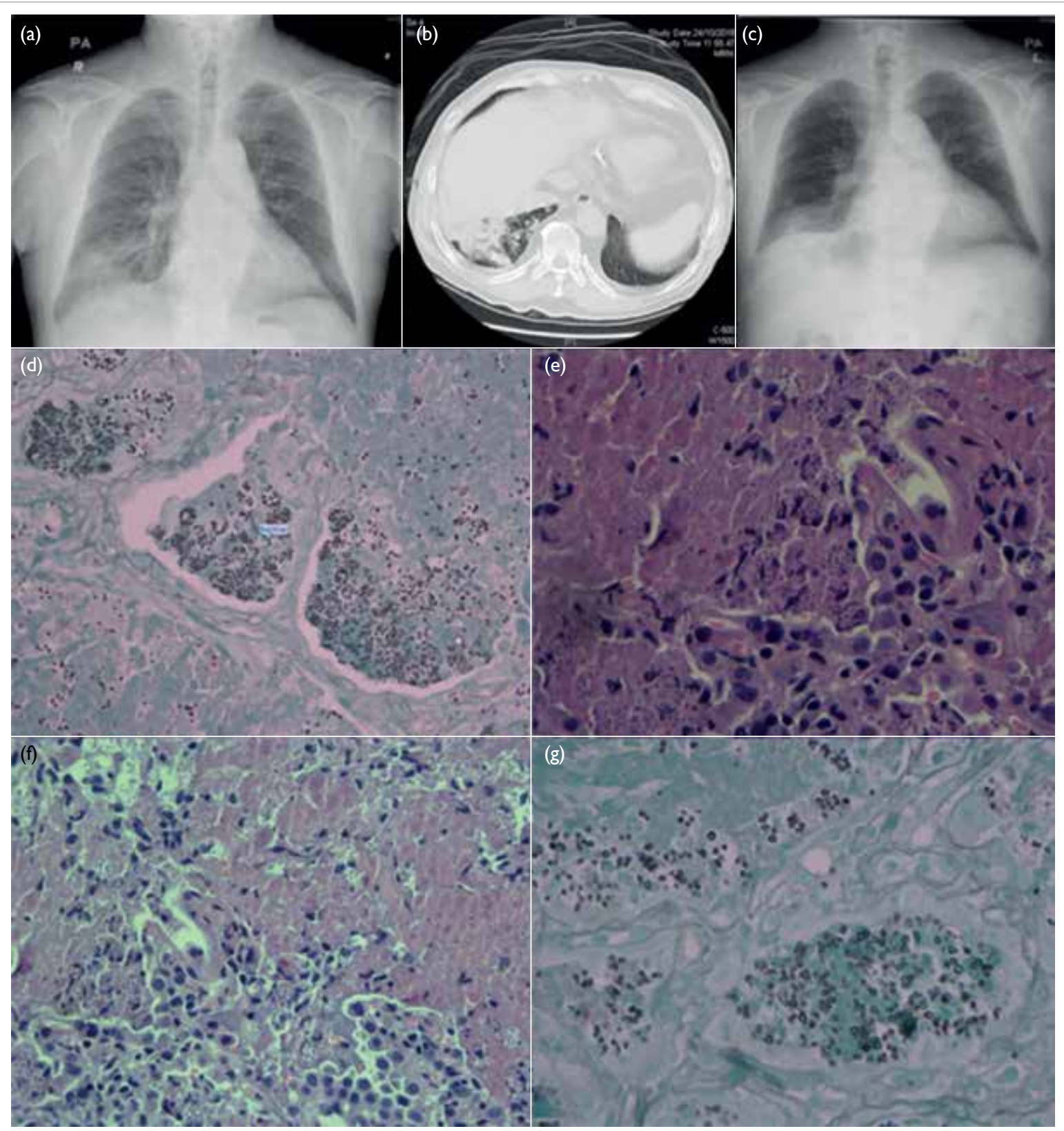

FIG I. (a) Initial chest X-ray on admission. (b) Computed tomographic thorax scan on day 16 after admission. (c) Chest $X$-ray after 2 weeks of antifungal therapy. (d) Gomori methenamine silver stain, $\times 400$, showing small yeasts of 3-4 $\mu \mathrm{m}$. (e) Haematoxylin and eosin stain, $\times 650$, showing intracellular yeasts. (f) Haematoxylin and eosin stain, $\times 400$, showing intracellular yeasts. (g) Gomori methenamine silver stain, $\times 650$

canadensis has been reported in Canada and the loss, and pulmonary disease. ${ }^{3,4}$ The diagnosis can United States. Emergomyces africanus has been be missed due to the slow-growing nature of the reported in South Africa, Emergomyces orientalis in fungus. Histological examination of tissues can China, and Emergomyces europaeus in Germany. The help diagnose the disease but on its own does not natural reservoir of Emergomyces is soil. Our patient distinguish infection from other dimorphic fungi. presumably acquired the infection via inhalation of Molecular diagnosis plays an important role in conidia in Hong Kong since he had no travel history microbiological investigation. Using broad-range outside of the region. fungal polymerase chain reaction to sequence D2 Emergomycosis is a multi-system disease. large-subunit rDNA gene can help to confirm the According to case reports, patients most often diagnosis in a rapid, sensitive, and specific way. present with fever, widespread skin lesions, weight

There are no treatment guidelines for patients 


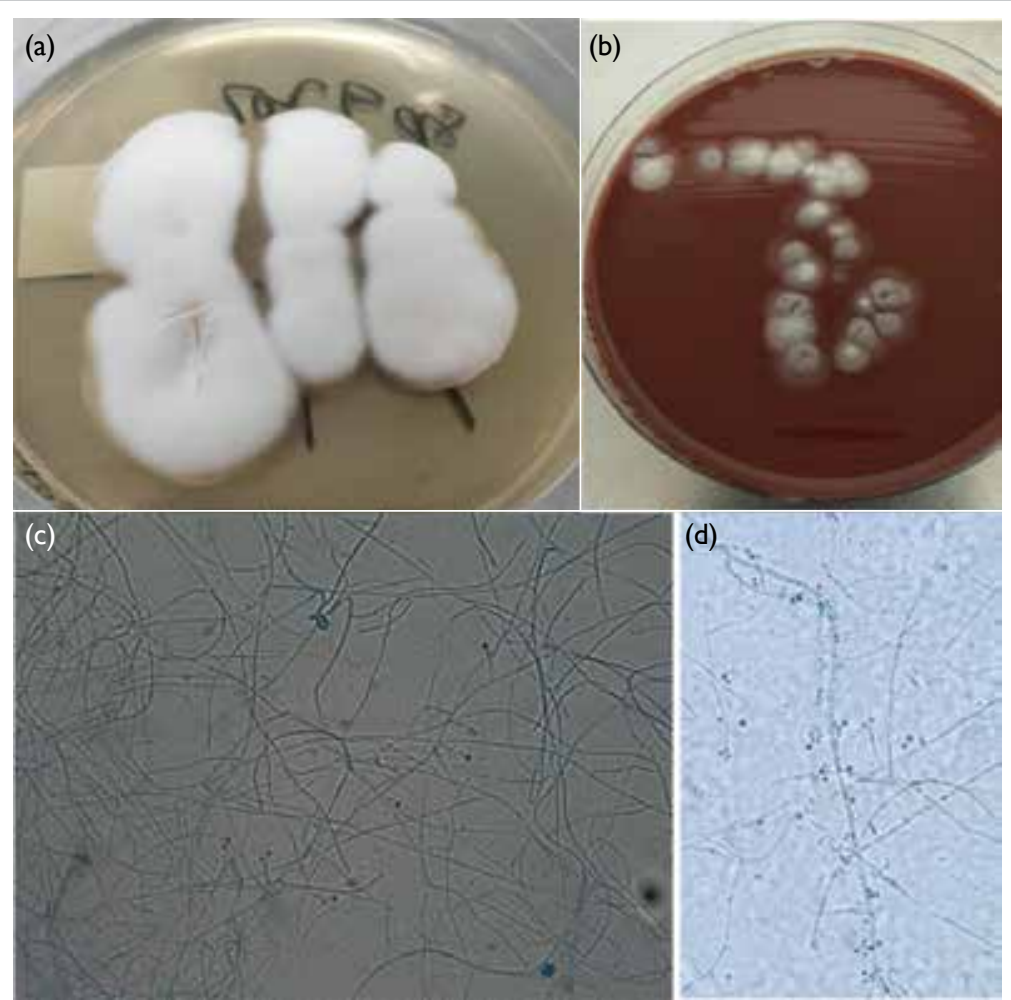

FIG 2. (a) Colonies on Sabouraud agar after 21 days incubation at $25^{\circ} \mathrm{C}$.

(b) Colonies on chocolate agar at day 21 of incubation at $25^{\circ} \mathrm{C}$. (c, d) Lactophenol cotton blue staining of the mould phase incubated at $25^{\circ} \mathrm{C}$ with emergomycosis. Guidelines for blastomycosis and histoplasmosis recommend liposomal amphotericin B as initial therapy followed by itraconazole (or other newer azole). Our patient responded to liposomal amphotericin $\mathrm{B}$ and oral voriconazole but passed away due to his medical disease.

More patients are now rendered immunosuppressed by advances in treatment for a variety of diseases. Clinicians and microbiologists should be aware of the presence of rare invasive fungal infections among these susceptible patients.
Molecular techniques such as internal transcribed spacer polymerase chain reaction and sequencing can aid early and accurate identification of these rare fungal pathogens.

\section{Author contributions}

All authors contributed to the concept or design of the study, acquisition of the data, analysis or interpretation of the data, drafting of the manuscript, and critical revision of the manuscript for important intellectual content. All authors had full access to the data, contributed to the study, approved the final version for publication, and take responsibility for its accuracy and integrity.

\section{Conflicts of interest}

The authors have no conflicts of interest to disclose.

\section{Acknowledgement}

The authors would like to thank pathologist Dr WL Lam for providing the histopathology clinical photos and nephrologist Dr SK Fung and Dr HL Tang for providing clinical information.

\section{Funding/support}

This case report received no specific grant from any funding agency in the public, commercial, or not-for-profit sectors.

\section{Ethics approval}

This case report was approved by the Hospital Authority Kowloon West Cluster Research Ethics Committee (Ref KW/ EX-19-063(137-04)).

\section{References}

1. Gast KB, van der Hoeven A, de Boer MG, et al. Two cases of Emergomyces pasteurianus infection in immunocompromised patients in the Netherlands. Med Mycol Case Rep 2019;24:5-8.

2. Koneru H, Penupolu S. Pulmonary adiaspiromycosis: an emerging fungal infection. Chest 2017;152 Suppl:A162.

3. Schwartz IS, Sanche S, Wiederhold NP, Patterson TF, Sigler L. Emergomyces canadensis, a dimorphic fungus causing fatal systemic human disease in North America. Emerg Infect Dis 2018;24:758-61.

4. Schwartz IS, Maphanga TG, Govender NP. Emergomyces: a new genus of dimorphic fungal pathogens causing disseminated disease among immunocompromised persons globally. Curr Fungal Infect Rep 2018;12:44-50. 